\title{
Woman With Post-Partum Kidney and Liver Failure
}

Joshua Gibbs M D , PG Y-3

The patient is a 34 year-old G IP1 C aucasian female with no medical history who was transferred from an outside hospital after an emergent C esarean section for increasing jaundice with elevated liver enzymes, abnormal coagulation times and acute renal failure. The patient was heal thy until 33 weeks gestation when she noted dizziness, headache and visual changes. Blood pressure at that time was elevated at $140 / 90$ and she had trace lower extremity edema. No proteinuria was detected. The clinical picture was consistent with mild pre-eclampsia. The patient was followed until 35 weeks gestation when she developed vaginal bleeding and abdominal pain. At that time, an emergency $\mathrm{C}$-section was performed due to a non-reassuring fetal heart rate. $0 \mathrm{n}$ the first night after the surgery, she became anuric and laboratory tests demonstrated acute renal failure, coagulopathy, and elevated liver enzymes. She became progressively jaundiced and she was transferred for further management. O n admission, she complained of nausea and malaise. She denied abdominal pain, chest pain, dyspnea, lightheadedness or headache.

Her past medical history was only significant for endometriosis. Her past surgical history included laparoscopic treatment of endometriosis several years ago and ovarian cyst removal several years ago. Her medications included Phenergan, Percocet, Lasix and Dulcolax. She had allergies to Augmentin and Ceclor which caused a rash. Her family history was noncontributory. The patient noted occasional tobacco use, denied alcohol or other substances. $\mathrm{H}$ er review of systems was only significant for mild dyspnea, nausea, mild gingival bleeding, and mild pruritis.

Vital signs showed a temperature of $99.0 \mathrm{~F}$, pulse of 84 beats per minute, respirations of 22 breaths per minute, blood pressure of 130/70 with blood oxygenation at 99\% on room air. Her physical examination was significant for scleral icterus; her heart rate was regular without murmurs, rubs or gallops; her lungs had bibasilar crackles; her abdomen was soft with some tenderness at her incision site with minimal oozing; she had moderate lochia and was heme negative rectally; she had $2+$ pitting edema on her bilateral lower extremities; neurologic examination was intact with some asterixis noted.

$\mathrm{H}$ er laboratory findings on admission were significant for a sodium of $122 \mathrm{mg} / \mathrm{dL}$, potassium of $5.2 \mathrm{mmol} / \mathrm{L}$, bicarbonate of $17 \mathrm{mmol} / \mathrm{L}$, BUN of $33 \mathrm{mg} / \mathrm{dL}$, creatinine of $3.3 \mathrm{mg} / \mathrm{dL}$, PTT of $55.6 \mathrm{sec}$, PT of $23 \mathrm{sec}$, IN R of 2.5 , fibrinogen of $94 \mathrm{mg} / \mathrm{dL}$, protein of $3.9 \mathrm{~g} / \mathrm{dL}$, albumin of $2.0 \mathrm{~g} / \mathrm{dL}$, total bilirubin of $9.7 \mathrm{mg} / \mathrm{dL}$, direct bilirubin of $5.4 \mathrm{mg} / \mathrm{dL}$, AST of $124 \mathrm{mg} / \mathrm{dL}$, ALT of $104 \mathrm{mg} / \mathrm{dL}$, alkaline phosphatate of $390 \mathrm{U} / \mathrm{L}$, ammonia of $57 \mathrm{~mol} / \mathrm{L}$, white blood cell count of $15.6 \mathrm{k} / \mathrm{L}$, hemoglobin of $9.6 \mathrm{~g} / \mathrm{dL}$, platelets of $174 \mathrm{k} / \mathrm{L}$, and a urinalysis with $3+$ protein.

Her chest X-ray showed mild pulmonary vascular congestion. A right upper quadrant ultrasound showed decreased flow in hepatic vein and mild ascites. $\mathrm{Her}$ $\mathrm{M} \mathrm{RI} / \mathrm{M} \mathrm{RA} / \mathrm{M} \mathrm{RV}$ of the abdomen showed narrowing of hepatic vein and inferior vena cava (IVC). The renal ultrasound showed no hydronephrosis.

\section{Discussion}

This case allows the discussion of liver disease during pregnancy, however the focus of this discussion will be on the Budd-C hiari Syndrome (BCS).

\section{Pathogenesis}

The patient in this case had signs and symptoms consistent with BCS or hepatic venous outflow obstruction. BCS describes a group of disorders that cause outflow obstruction at the level of the hepatic venule, large hepatic veins, inferior vena cava or right atrium. $\mathrm{H}$ epatic veno-occlusive disease refers to obstruction at the level of the center of sub-lobular hepatic veins. $\mathrm{H}$ epatic venous outflow tract obstruction results in increased sinusoidal pressure and portal hypertension. Early in the disease, portal venous perfusion is diminished which may result in portal vein thrombosis. Venous stasis leads to centrolobular necrosis, fibrosis and eventually cirrhosis. If a portosystemic shunt is created or portal venous collaterals develop, liver function improves by reducing sinusoidal pressure. About $75 \%$ of patients have an identifiable cause of BCS. Hereditary and acquired hypercoagulable states are a common cause. $M$ yeloproliferative disorders are the most common cause of BCS, most notably polycythemia vera. 0 ther causes include paroxysmal nocturnal hemoglobinuria, antiphospholipid syndrome, inherited deficiencies of proteins $C$ and $S$ and antithrombin III. BCS has been described in pregnancy and the immediate post-partum period.

\section{Clinical M anifestations}

BCS can be grouped into fulminant, acute, subacute and chronic depending on the speed which the hepatic veins become occluded and whether collateral circulation 
exists. Abdominal pain, hepatomegaly and ascites are the most common findings. N ausea, vomiting and jaundice are more common in the fulminant and acute forms. Splenomegaly and esophageal varices are more common in the chronic forms. IVC occlusion usually causes dilated venous collaterals on the back and flanks. Patients with acute BCS have ascites, hepatic necrosis, symptoms of short duration and lack of venous collaterals. H epatic necrosis and ascites are usually minimal in the subacute form because venous collaterals have decompressed the hepatic sinusoids. The subacute form is the most common form of BCS. A careful cardiovascular examination can separate BC S from cardiac causes of diminished hepatic venous outflow. Right atrial myxoma, tricuspid regurgitation and constrictive pericarditis can cause similar symptoms to BCS. Lack of hepatojugular reflux is indicative of BCS on physical examination.

\section{D iagnosis}

Laboratory evaluation shows AST and ALT to be five times the upper limit of normal in fulminant and acute forms of BCS. The subacute form has lower values. Alkaline phosphatase and bilirubin levels are also elevated. Serum-ascitic fluid albumin gradient is high. D oppler ultrasound is the diagnostic imaging of choice with a sensitivity and specificity of $85 \%$. M RI can show hepatic vein thrombosis and may be better for delineating acute from subacute and chronic forms. $H$ epatic venography is used as a confirmatory test when ultrasound is negative but there is a strong clinical suspicion. The classic "spiderweb" pattern is often seen. The patient in this case had an M RI which showed narrowing of the hepatic vein and IVC which is consistent with $\mathrm{BCS}$.

\section{M anagement}

$M$ edical management focuses on treating the underlying cause, anticoagulation to prevent extension of thrombosis and efforts to reduce ascites. D iuretics such as furosemide and spironolactone as well as paracentesis and sodium restriction are used to reduce ascites. $\mathrm{H}$ eparin is used initially with conversion to warfarin with a goal INR of 2.0 to 2.5. Medical management is generally recommended for patients with few symptoms, no hepatic necrosis and minimal ascites. Patients with coagulopathy, encephalopathy and hepatorenal syndrome have a poor prognosis and require immediate relief of hepatic venous obstruction.
Thrombolytics can be used in the acute form of BCS particularly when an acute thrombus is seen on angiography. TPA or urokinase is directly infused by catheter for 24 hours. The overall success rate is low with thrombolytics, but positive results have occurred as far as two to three weeks after onset of symptoms. Percutaneous or transhepatic angioplasty of segments of the narrowed hepatic vein can also be performed. Symptom relief occurs in up to $70 \%$ of patients, but restenosis rates are high.

Transjugular intrahepatic portosystemic shunts (TIPS) are typically used in patients who have an occluded IVC when the portal vein-infrahepatic vena caval pressure gradient is less than $10 \mathrm{~mm} \mathrm{H} \mathrm{g}$ and when there is poor hepatic reserve. TIPS can al so be used in acute BCS after failure of thrombolytic therapy. TIPS can be used as a bridge to liver transplantation. Even if the shunt occludes, it may allow enough time for collaterals to develop and decrease sinusoidal pressure.

Surgical options include excision of IVC webs followed by angioplasty of the vena cava, although portosystemic shunting and liver transplantation are the most common surgical treatments for BCS. Surgical portosystemic shunts are recommended in subacute forms of BCS when the underlying etiology has a favorable outcome, the patient is a good surgical candidate and when liver biopsy shows ongoing hepatic necrosis. A portal vein and IVC pressure gradient of more than $10 \mathrm{~mm} \mathrm{Hg}$ is associated with a successful long-term outcome. Examples of surgical shunts include side-to-side portocaval shunt, central splenorenal shunt, and a mesocaval shunt. The five-year survival rate after surgical shunting is between $75 \%$ and $94 \%$.

The five-year survival rate for liver transplantation in patients with BCS is $95 \%$. Indications are an underlying disease associated with a favorable prognosis, fulminant hepatic failure, cirrhosis, and failure of portosystemic shunt. $\mathrm{N}$ ot all patients require long-term anticoagulant therapy after liver transplantation, but it may be reasonable to anticoagulate since patients with BCS have multiple underlying etiologic factors.

\section{References}

1. M enon KV, Shah V, Kamath PS. The Budd-Chiari Syndrome. N Engl J M ed 2004; 350(6): 578-585. 